\title{
Analyzing Classifier Hierarchy Multiclassifier Learning
}

\author{
J.M. Martínez-Otzeta ${ }^{1}$, B. Sierra ${ }^{2}$, E. Lazkano ${ }^{2}$, E. Jauregi ${ }^{2}$, \\ and Y. Yurramendi ${ }^{2}$ \\ ${ }^{1}$ Fundación Tekniker \\ Otaola 20, 20600 Eibar, Basque Country, Spain \\ jmmartinez@tekniker.es \\ ${ }^{2}$ Department of Computer Science and Artificial Intelligence \\ University of the Basque Country \\ P. Manuel Lardizabal 1, 20018 Donostia-San Sebastián \\ Basque Country, Spain \\ b.sierra@ehu.es
}

\begin{abstract}
Classifier combination falls in the so called machine learning area. Its aim is to combine some classification paradigms in order to improve the individual accuracy of the component classifiers. Classifier hierarchies are an alternative among the several methods of classifier combination. In this paper we present new results about a recently proposed hierarchy construction method. Experiments have been carried out over 42 databases from the UCI repository, showing an improvement over the performance of the base classifiers.
\end{abstract}

Keywords: Classifier combination, hierarchy of classifiers, data mining.

\section{Introduction}

In the last years different approaches to improve the performance of a single classifier have been studied in the machine learning community, being one of them the combination of classifiers. There are very different manners to combine classifiers, most of which have been found to improve accuracy over single classifiers 3]. Several approaches have been studied, among them: bagging [5], that uses more than one model of the same paradigm in order to reduce errors; boosting [6], in which a different weight is given to different training examples looking for a better accuracy; bi-layer classifiers [4 where several models from different paradigms are combined in a parallel mode, using the individual decision of each one as predictor variable for a new classifier which takes the final decision; there are some other combination approach in serial or semi-parallel architectures 7 . Other way is to build a classifier hierarchy, constructed in the shape of a tree. This hierarchy could be learned in a recursive mode, using a top-down approach to select the classifier of each of the edges, taking into account the data.

In this paper we continue the work presented in [8], where the authors define a new hierarchy construction method and make a search in the space of all possible 
hierarchies by means of a genetic algorithm. Here we show that good results could also be achieved by a pure random search. Our results have been obtained over 42 databases from the UCI repository, three times as much databases than in the original paper, where only 14 were analyzed.

The rest of the paper is organized as follows: Section 2 gives a brief introduction to the hierarchical model of combination of classifiers. In section 3 how to construct an actual model from a hierarchy of classifiers is described. Section 4 is devoted to the experimental setup and results obtained, while the final section presents the conclusions and points out the future work.

\section{Hierarchy of Classifiers}

As mentioned above, the combination of multiple classifiers has been intensively studied with the aim of improving the individual accuracy of individual components. It has been shown that in many domains an ensemble of classifiers outperforms any of its single components.

According to the architecture used to combine different single classifiers, Lu 11] cites three possible configurations: cascaded, parallel and hierarchical. The characteristics of these architectures are:

- In a cascaded system, the classification results generated by a classifier are often used to direct the classification processes of successive classifiers. The main problem in this method is that errors made by previous classifiers are not recoverable by the successive classifiers.

- In a parallel system, there is a true integration of knowledge of different sources, generated independently. If the decision process is well designed, the overall system may reach peak performance.

- In a hierarchical system, the control strategy is a combination of cascaded and parallel processing.

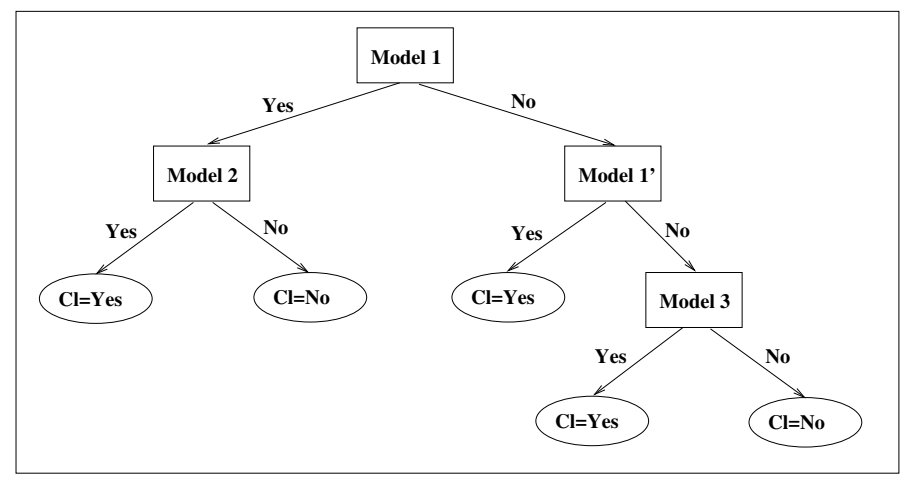

Fig. 1. A hierarchy of classifiers in a Yes-No problem. In this example there are three different classifiers that induce three kinds of models (Model 1 to Model 3). 
The approach analyzed in this paper falls within the hierarchical model [12].

A possibility to form this hierarchy is to arrange the single classifiers in a tree. In such a representation, a new case runs the following path: starting at the root node, the instance is classified according to the model associated to that node; then a test is performed on the outcome of the model and, according to the result, a branch is selected and the process continues until the case reaches a leaf, and the outcome of the model at this stage is returned. See Figure 1 for an example.

The idea behind this procedure is that the mistakes a classifier makes can be the input to another classifier, in such a way that this one could discover some patterns in the mistakes. Iteration of this procedure leads to a hierarchy (or tree) of classifiers.

\section{Induction of Individual Classifiers in a Single Hierarchy}

Given a hierarchy schemata, the classifiers corresponding to each node should be induced. To do that, we apply a recursive algorithm similar to the so called Top Down Induction of Decision Trees [14] as shown in Figure 2.

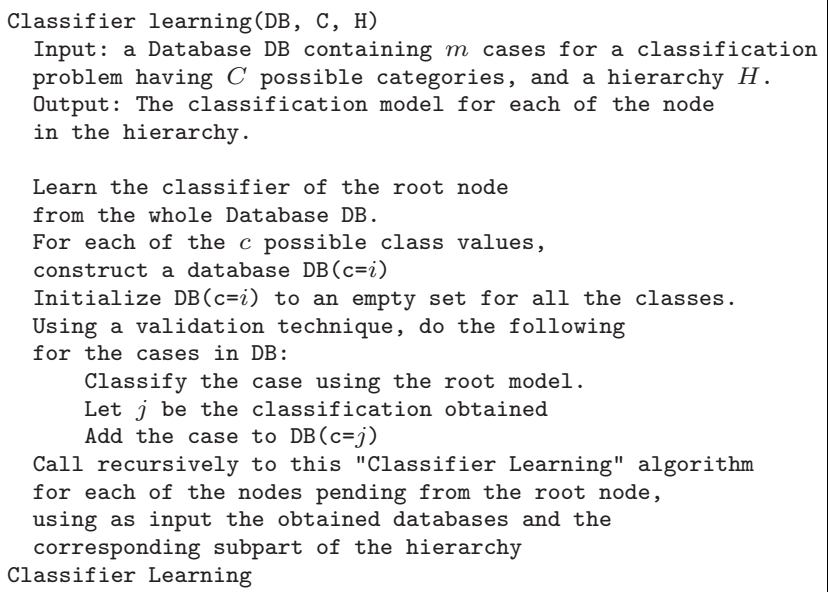

Fig. 2. Classifier induction process for a given hierarchy

The following example will help to understand this process.

Given the hierarchy in Figure 1 and supposing that the hierarchy is built with three classifier paradigms $(I=3)$, for a two-class problem. Let us denote the two classes as Yes, No. It is necessary to build the classifier model for each of the nodes in the tree that forms the hierarchy. Let us name Example.data the datafile where all the available instances corresponding to our classification problem are collected. The procedure is as follows: 
- The classifier in the root node of the hierarchy is constructed using all the cases in Example.data

- The classifier in every node different from the root is constructed in the following way:

- Get the database which was used to build the classifier at the parent node.

- Divide the database into $n$ folds of the same size.

- For each fold, classify each case in the fold using the model induced by the remaining $n-1$ folds.

- If class of case = yes, add it to Exampleyes.data else add it to Exampleno.data.

- Induce the corresponding models using the obtained databases.

- If the node is reached from the parent node through a Yes edge, then use Exampleyes.data to build the model associated at that node. If it is not (that is, if the node is reached through a No edge), use Exampleno.data to build the model.

- If all the cases are classified as Yes or No, create a leaf at that node.

In our setup the number of folds is 7 , according to the results shown in 9 .

\section{Experimental Setup and Results}

In order to evaluate the performance of the proposed approach, we have carried out an experiment over 42 databases from the UCI repository [13. In our experimental setup we have chosen four base classifiers: ID3 [14, C4.5 [16], Naive Bayes [15] and IB1 [1], available in $\mathcal{M L C}++$ libraries [2].

The criterion to define the fitness of a hierarchy is the performance of a $5 \times 2$ cross validation over each database [10. In Table 1 are presented the accuracies of the four base classifiers. In boldface is shown the best of all four.

As a first approach, we have chosen 200 random three-level hierarchies, as well as 500 random four-level hierarchies. In Table 2 the best accuracy obtained by a hierarchy is shown under the label Best depth 3 and Best depth 4, for hierarchies of three and four levels respectively. In Relative best base the number of hierarchies found better than the best of the base classifiers is shown under the Wins label, along with the number of hierarchies that tie, under the Ties label. From these tables it is observed that, when restricted to hierarchies of three levels, a best results than the best case classifier is obtained in 23 out of 42 databases, while there are 16 ties and only in three cases the best hierarchy performs worse than the best base classifier. When evaluating the hierarchies of four levels, the results are similar, though a bit better $(+23=18-1)$.

Other experiment has been carried out, this time evaluating all possible twolevel hierarchies in the 26 two-classes problems out of the 42 original databases. As there are four different possibilities in the root node (the four base classifiers) and five in each of the two branches corresponding to each class (the four base 
Table 1. Accuracies over the databases used in this experiment

\begin{tabular}{|c|c|c|c|c|}
\hline Database & $\overline{N B}$ & $\overline{I I b}$ & $\overline{I I d 3}$ & $C 4.5$ \\
\hline Anneal & 93.01 & 97.35 & 98.57 & 98.13 \\
\hline Australian & 76.84 & 80.96 & 80.96 & 84.58 \\
\hline Auto & 55.13 & 65.26 & 66.24 & 62.34 \\
\hline Balance Scale & 89.95 & 82.30 & 78.18 & 78.98 \\
\hline Banding & 75.71 & 76.05 & 74.20 & 78.24 \\
\hline Breast & 95.91 & 95.65 & 94.42 & 94.31 \\
\hline Cars & 98.88 & 98.98 & 96.89 & 96.38 \\
\hline Chess & 87.15 & 92.87 & 98.87 & 99.00 \\
\hline Churn & 88.57 & 83.52 & 91.54 & 94.12 \\
\hline Cleve & 82.97 & 77.49 & 73.00 & 76.97 \\
\hline Corral & 83.91 & 91.09 & 96.41 & 92.19 \\
\hline Crx & 77.45 & 81.57 & 80.70 & 84.96 \\
\hline Diabetes & 75.31 & 69.66 & 69.43 & 72.71 \\
\hline Echocardiogram & 71.90 & 56.18 & 61.82 & 63.96 \\
\hline Flare & 80.88 & 80.88 & 80.69 & 82.33 \\
\hline German & 74.46 & 69.22 & 69.08 & 70.92 \\
\hline Glass & 50.28 & 64.95 & 64.95 & 64.21 \\
\hline Glass2 & 62.58 & 76.81 & 77.28 & 76.08 \\
\hline Hayes Roth & 70.62 & 63.62 & 84.37 & 83.75 \\
\hline Heart & 82.30 & 74.44 & 74.74 & 75.56 \\
\hline Hepatitis & 85.40 & 80.38 & 74.84 & 78.31 \\
\hline Horse-colic & 78.97 & 73.64 & 78.42 & 85.54 \\
\hline Hungarian & 84.22 & 79.39 & 77.21 & 79.86 \\
\hline Hypothyroid & 97.80 & 96.96 & 98.62 & 99.15 \\
\hline Iris & 95.47 & 94.93 & 94.67 & 94.13 \\
\hline Lenses & 64.17 & 69.17 & 75.00 & 69.17 \\
\hline Liver-disorder & 56.46 & 59.13 & 60.17 & 59.94 \\
\hline Lymphography & 78.38 & 79.73 & 76.62 & 78.92 \\
\hline Mushroom & 99.67 & 100.00 & 99.98 & 99.98 \\
\hline Parity $5+5$ & 47.05 & 51.05 & 63.87 & 53.71 \\
\hline Pima & 75.39 & 69.24 & 69.56 & 71.59 \\
\hline Solar & 66.13 & 65.63 & 68.36 & 71.64 \\
\hline Sonar & 68.85 & 83.08 & 72.98 & 70.96 \\
\hline Splice & 95.22 & 72.51 & 89.40 & 92.52 \\
\hline ThreeOf9 & 79.53 & 90.62 & 96.60 & 95.00 \\
\hline Tic-tac-toe & 70.31 & 95.64 & 82.42 & 81.38 \\
\hline Tokyo1 & 88.53 & 91.43 & 89.72 & 91.16 \\
\hline Vehicle & 45.41 & 68.13 & 69.67 & 70.50 \\
\hline Vote & 90.20 & 92.55 & 93.52 & 95.08 \\
\hline Vote1 & 87.17 & 89.52 & 87.08 & 87.95 \\
\hline Wine & 96.74 & 94.49 & 88.76 & 87.75 \\
\hline Zoo & 80.03 & 94.26 & 92.48 & 90.32 \\
\hline
\end{tabular}


Table 2. Accuracy of the best hierarchy along with the number of hierarchies outperforming it for several depths

\begin{tabular}{|c|c|c|c|c|c|c|}
\hline \multirow{3}{*}{ Database } & \multicolumn{3}{|c|}{ Depth 3} & \multicolumn{3}{|c|}{ Depth 4} \\
\hline & \multirow[t]{2}{*}{ Best accuracy } & \multicolumn{2}{|c|}{ Relative best base } & \multirow[t]{2}{*}{ Best accuracy } & \multicolumn{2}{|c|}{ Relative best base } \\
\hline & & \begin{tabular}{|l|} 
Wins \\
\end{tabular} & Ties & & \begin{tabular}{|l|} 
Wins \\
\end{tabular} & Ties \\
\hline Anneal & 98.73 & 6 & 4 & 98.73 & 12 & 13 \\
\hline Australian & 84.93 & 4 & 0 & 85.01 & 8 & 4 \\
\hline Auto & $64.78^{*}$ & 0 & 0 & $65.95^{*}$ & 0 & 0 \\
\hline Balance Scale & 89.95 & 0 & 2 & 89.95 & 0 & 4 \\
\hline Banding & 79.41 & 3 & 0 & 78.82 & 3 & 0 \\
\hline Breast & 96.65 & 12 & 0 & 96.68 & 39 & 3 \\
\hline Cars & 99.18 & 36 & 48 & 99.18 & 70 & 128 \\
\hline Chess & 99.07 & 16 & 1 & 99.14 & 11 & 6 \\
\hline Churn & 94.12 & 0 & 4 & 94.12 & 0 & 3 \\
\hline Cleve & 83.04 & 2 & 1 & 82.97 & 0 & 10 \\
\hline Corral & 96.41 & 0 & 17 & 96.41 & 0 & 31 \\
\hline Crx & 85.54 & 7 & 1 & 85.30 & 6 & 7 \\
\hline Diabetes & 75.31 & 0 & 12 & 75.70 & 1 & 5 \\
\hline Echocardiogram & 71.90 & 0 & 4 & 71.90 & 0 & 9 \\
\hline Flare & 82.70 & 17 & 0 & 82.81 & 56 & 10 \\
\hline German & 74.60 & 2 & 1 & 74.64 & 2 & 5 \\
\hline Glass & 65.98 & 2 & 0 & 65.14 & 1 & 0 \\
\hline Glass2 & 78.65 & 8 & 0 & 78.41 & 12 & 3 \\
\hline Hayes Roth & 84.63 & 3 & 0 & 84.88 & 11 & 0 \\
\hline Heart & 82.30 & 0 & 2 & 82.30 & 0 & 5 \\
\hline Hepatitis & 85.40 & 0 & 3 & 85.40 & 0 & 4 \\
\hline Horse-colic & 85.54 & 0 & 1 & 85.54 & 0 & 4 \\
\hline Hungarian & 84.22 & 0 & 3 & 84.22 & 0 & 4 \\
\hline Hypothyroid & 99.15 & 0 & 2 & 99.15 & 0 & 8 \\
\hline Iris & 96.00 & 11 & 10 & 96.00 & 11 & 12 \\
\hline Lenses & 75.00 & 0 & 16 & 75.00 & 0 & 63 \\
\hline Liver-disorder & 63.83 & 74 & 1 & 63.65 & 173 & 10 \\
\hline Lymphography & 80.68 & 7 & 3 & 81.35 & 22 & 8 \\
\hline Mushroom & 100.00 & 0 & 58 & 100.00 & 0 & 132 \\
\hline Parity $5+5$ & 63.87 & 0 & 3 & 63.87 & 0 & 2 \\
\hline Pima & 75.52 & 1 & 0 & 75.96 & 2 & 8 \\
\hline Solar & $71.51^{*}$ & 0 & 0 & 71.64 & 0 & 5 \\
\hline Sonar & 83.08 & 0 & 2 & 83.08 & 0 & 5 \\
\hline Splice & 95.26 & 2 & 1 & 95.26 & 4 & 1 \\
\hline ThreeOf9 & 96.64 & 2 & 3 & 96.68 & 3 & 6 \\
\hline Tic-tac-toe & 95.74 & 5 & 36 & 95.82 & 5 & 75 \\
\hline Tokyo1 & 91.93 & 9 & 0 & 91.95 & 18 & 1 \\
\hline Vehicle & $70.35^{*}$ & 0 & 0 & 70.50 & 0 & 1 \\
\hline Vote & 95.40 & 9 & 2 & 95.54 & 22 & 9 \\
\hline Vote1 & 89.79 & 2 & 3 & 89.98 & 4 & 7 \\
\hline Wine & 96.74 & 0 & 43 & 96.74 & 0 & 132 \\
\hline Zoo & 94.26 & 0 & 49 & 94.26 & 0 & 129 \\
\hline
\end{tabular}


Table 3. Results over the 26 two-class databases using two level classifiers

\begin{tabular}{|l|r|r|r|}
\hline \hline Database & Best result & Root classifier & Best \\
\hline \hline Australian & 84.43 & $\mathrm{c} 4.5$ & $\mathrm{c} 4.5(84.58)$ \\
Banding & 78.74 & $\mathrm{nb}$ & $\mathrm{c} 4.5(78.24)$ \\
Breast & 96.68 & $\mathrm{ib}$ & $\mathrm{ib}(95.91)$ \\
Chess & 99.07 & $\mathrm{c} 4.5$ & $\mathrm{c} 4.5(99.00)$ \\
Churn & 94.10 & $\mathrm{c} 4.5$ & $\mathrm{c} 4.5(94.12)$ \\
Cleve & 82.77 & $\mathrm{nb}$ & $\mathrm{nb}(82.97)$ \\
Corral & 96.41 & $\mathrm{id} 3$ & $\mathrm{id} 3(96.41)$ \\
Crx & 85.65 & $\mathrm{id} 3$ & $\mathrm{c} 4.5(84.96)$ \\
Diabetes & 75.08 & $\mathrm{nb}$ & $\mathrm{nb}(75.31)$ \\
Echocardiogram & 71.44 & $\mathrm{c} 4.5$ & $\mathrm{nb}(71.90)$ \\
Flare & 82.66 & $\mathrm{c} 4.5$ & $\mathrm{ib}(82.33)$ \\
German & 74.60 & $\mathrm{nb}$ & $\mathrm{nb}(74.46)$ \\
Glass2 & 78.17 & $\mathrm{c} 4.5$ & $\mathrm{id} 3(77.28)$ \\
Heart & 80.96 & $\mathrm{nb}$ & $\mathrm{nb}(82.30)$ \\
Hepatitis & 84.49 & $\mathrm{nb}$ & $\mathrm{nb}(85.40)$ \\
Horse-colic & 85.38 & $\mathrm{c} 4.5$ & $\mathrm{c} 4.5(85.54)$ \\
Hungarian & 83.67 & $\mathrm{nb}$ & $\mathrm{nb}(84.22)$ \\
Hypothyroid & 99.15 & $\mathrm{c} 4.5$ & $\mathrm{c} 4.5(99.15)$ \\
Liver-disorder & 63.65 & $\mathrm{id} 3$ & $\mathrm{nb}(60.17)$ \\
Mushroom & 100.00 & $\mathrm{ib}$ & $\mathrm{ib}(100.00)$ \\
Pima & 75.34 & $\mathrm{nb}$ & $\mathrm{nb}(75.39)$ \\
Sonar & 82.40 & $\mathrm{ib}$ & $\mathrm{ib}(83.08)$ \\
Tic-tac-toe & 95.64 & $\mathrm{ib}$ & $\mathrm{ib}(95.64)$ \\
Tokyo1 & 91.91 & $\mathrm{c} 4.5$ & $\mathrm{nb}(91.43)$ \\
Vote & 95.40 & $\mathrm{c} 4.5$ & $\mathrm{c} 4.5(95.08)$ \\
Vote1 & 89.61 & $\mathrm{nb}$ & $\mathrm{ib}(89.52)$ \\
\hline \hline
\end{tabular}

classifiers and the possibility of being a leaf), a total of $5 \times 5 \times 4=100$ hierarchies have been studied. Results are shown in Table 3. Best result stands for the best result of a no-simple hierarchy. Root classifier is the classifier at the root node of the previously cited hierarchy. And Best base classifier is the best performing among the base classifier along with its accuracy. From this table we observe that the root node of the best no-simple classifier matches the best base classifier in 17 out of 26 databases, suggesting that a greedy search could be of interest, instead of simply a random search or something more demanding as a genetic search. It has also to be noticed that, even in the cases (9 out of 26 databases) where the root classifier in the best hierarchy does not match with the best base classifier, there are other hierarchies which outperform that best single classifier, using that classifier as root node.

\section{Conclusions and Future Work}

New results about a recently proposed hierarchy construction method have been presented in this paper. Experiments have been carried out over 42 databases 
from the UCI repository, with hierarchies of two, three and four levels. Our results with a random search over three and four levels suggest that it could be possible to implement a greedy search instead of a genetic search. The small improvement obtained with four levels over the three levels hierarchies suggest that three could be the ideal number of levels in a hierarchy. Experiments over two level hierarchies show that usually the root classifier in the best no-simple hierarchy matches with the best base classifier, suggesting the convenience of starting the greedy search with the best base classifier as root node of the hierarchy.

Future work involves more detailed research on greedy search, with problems with more of two classes, as well with three or more level hierarchies. Research about the best number of levels of hierarchies are going to be conducted too.

\section{References}

1. Aha, D., Kibler, D., Albert, M.K.: Instance-Based learning algorithms. Machine Learning 6, 37-66 (1991)

2. Kohavi, R., Sommerfield, D., Dougherty, J.: Data Mining Using MLC++: A Machine Learning Library in $\mathrm{C}++$. International Journal on Artificial Intelligence Tools 6(4), 537-566 (1997)

3. Ho, T.K., Hull, J.J., Srihari, S.N.: Decision Combination in Multiple Classifier Systems. IEEE Transactions on Pattern Analysis and Machine Intelligence 16(1), 66-75 (1994)

4. Wolpert, D.H.: Stacked Generalization. Neural Networks 5, 241-259 (1992)

5. Breiman, L.: Bagging predictors. Machine Learning 24(2), 123-140 (1996)

6. Freund, Y., Schapire, R.E.: A Short Introduction to Boosting. Journal of Japanese Society for Artificial Intelligence 14(5), 771-780 (1999)

7. Gama, J.M.: Combining Classification Algorithms. PhD Thesis, Universidade do Porto (2000)

8. Martínez-Otzeta, J.M., Sierra, B., Lazkano, E., Astigarraga, A.: Classifier Hierarchy Learning by means of Genetic Algorithms. Pattern Recognition Letters 27(16), 1998-2004 (2006)

9. Martínez-Otzeta, J.M., Sierra, B., Lazkano, E., Jauregi, E., Yurramendi, Y.: Naive + Naive $=$ Smart Bayes? In: ICARA 2006: 3rd International Conference on Autonomous Robots and Agents, vol. I, pp. 291-295. Massey University Press, New Zealand (2006)

10. Demšar, J.: Statistical Comparisons of Classifiers over Multiple Data Sets. Journal of Machine Learning Research 7, 1-30 (2006)

11. Lu, Y.: Knowledge Integration in a Multiple Classifier System. Applied Intelligence 6(2), 75-86 (1996)

12. Jordan, M.I., Jacobs, R.A.: Hierarchical Mixtures of Experts and the EM Algorithm. Neural Computation 6(2), 181-214 (1994)

13. University of California, Irvine, School of Information and Computer Sciences, http://www.ics.uci.edu/ mlearn/MLRepository.html

14. Quinlan, J.R.: Induction of Decision Trees. Machine Learning 1, 81-106 (1986)

15. Minsky, M.: Steps toward Artificial Intelligence. Proceedings of the Institute of Radio Engineers 49, 8-30 (1961)

16. Quinlan, J.R.: C4.5: Programs for Machine Learning. Morgan Kaufmann, San Mateo (1993) 